\title{
Analysis on acceptance of elderly drivers for intelligent speed adaptation using a driving simulator
}

\author{
R. Ando \& Y. Mimura \\ Research Department, \\ TTRI (Toyota Transportation Research Institute), Japan
}

\begin{abstract}
This paper aims to make a discussion on acceptability of elderly drivers for Intelligent Speed Adaptation (ISA) in Japan. In Japan, the issue focusing on the traffic safety of elderly people has become one of most important issues because Japan has been the most representative country to be the rapidly ageing society. As a measure of traffic safety, the ISA is considered as an effective measure to reduce the number of traffic accidents in the field of ITS (Intelligent Transportation Systems) because the adaptable driving speeds let the traffic flow smoothly and stably. Generally, there are three modes regarding the ISA: 1. advisory mode, in which the driving speed information is provided to the driver as the system detects that the vehicle is moving beyond the enforced speed limit; 2 . mandatory mode, by which the driving speed is regulated by the ISA so as not to exceed the allowable speed; 3 . voluntary mode, which extricate the mandatory mode and grant the driver full control of the vehicle's acceleration. Normally, the mandatory type is the most effective one but is difficult to introduce because of too many obstacles such as the freedom of personal choice, the limitation for the automobile technology and so on. Comparatively the advisory type seems to be the easiest one to introduce. The analysis is based on an experiment by using a driving simulator. The targeted issues focus on the acceptances of both advisory mode and mandatory mode for the elderly driver because grasping of the acceptances for the ISA is absolutely essential to implement the novel system for the ageing society of Japan. As the conclusion of the paper, the acceptances of the elderly drivers for both mandatory ISA and advisory ISA on the community roads are generally high.

Keywords: elderly drivers, ISA (Intelligent Speed Adaptation), driving simulator.
\end{abstract}




\section{Introduction}

Traffic fatalities have been on the decline for 12 consecutive years in Japan. On the other hand, the percentage of the fatalities in the community streets, in which the road way width is narrower than $5.5 \mathrm{~m}$, and the elderly people, who are 65 or more, are increasing [1]. The speed of vehicle at the time of collision is greatly related damage of traffic accidents [2]. Therefore, reducing the speeds is considered as an expectable measure [3].

On the other hand, Japan has been the most representative country to be the rapidly ageing society in the World. Regarding the traffic safety for the elderly people, the issue may be discussed in both viewpoints being a victim in traffic accidents or an offender who caused the traffic accidents. In this paper, we focus on the latter viewpoint to discuss how to make the drive speed slow down so that the traffic accidents resulting in injury may be decreased.

The Ninth Fundamental Traffic Safety Program of Japan was released in 2011 [1]. One concrete counter measure is to introduce Zone 30 widely in Japan. To make Zone 30 really functional, the Intelligent Speed Adaptation (ISA), which is one of the ITS (Intelligent Transportation Systems) technologies controlling vehicle speed by means of an in-vehicle system, is considered to be effective [3].

Generally, the ISA is classified by three modes: 1 . advisory mode, in which the driving speed information is provided to the driver as the system detects that the vehicle is moving beyond the enforced speed limit; 2 . mandatory mode, by which the driving speed is regulated by the ISA so as not to exceed the allowable speed; 3. voluntary mode, which extricate the mandatory mode and grant the driver full control of the vehicle's acceleration. Normally, the mandatory type is the most effective one but is difficult to introduce because of too many obstacles such as the freedom of personal choice, the limitation for the automobile technology and so on. Comparatively, the advisory type seems to be the easiest one to introduce.

We believe that if the verification of the system effects were done properly, the ISA will become innovative measures [4-6] to guarantee the effectiveness of the speed limit in the community streets in Japan. Furthermore, considering primarily the acceptances of the ISA for elderly driver is absolutely essential to implement the novel system for the aging society of Japan.

This study aims to determine the acceptability of the elderly driver of the ISA. The targeted issues focus on both advisory mode and mandatory mode.

\section{Review on previous studies}

The review can be made from two respects related with this study. One is on the characteristics of the elderly drivers being different with the other drivers. Another viewpoint is on the ISA applications.

The previous studies focusing on the elderly drivers' characteristics are simply reviewed in terms of Committee of the Elderly Drivers' Driving Aptitude of Chubu Branch [7] and International Association of Traffic and Safety Science [8]. The general understanding up to now in the World are widely common. The decrease of the physical ability related with the driving ability is mainly because 
the decrease of the eyesight, hearing ability and reaction velocity. The other reasons causing the decrease of the driving ability are from the psychological factors such as the elderly drivers tend to behave self-centeredness or egotism.

In addition to the above mentioned papers, the review on the ISA applications are on the basis of special issue of Accident Analysis \& Prevention with papers by Carsten [9], Lai and Carsten [10], Lahrmann et al. [11, 12], Chorlton et al. [13], van der Pas et al. [14] and Young et al. [15]. The ISA applications have been widely studied in Europe and the World. All three modes of the ISA have been concluded effective to reduce the speeds. Hinted from these studies, the ISA services should be examined to be introduced into Japan as our main target is to reduce the speeds. This is the one important purpose to do this study.

Meanwhile, the ISA may help the lots of elderly drivers who have problems both of the physical and psychological aspects mentioned above, because the novel system supports recognizing the current situation, making the correct judgments so that making the correct operation while driving. Therefore we thought that a comparative analysis by the age groups should be carried out.

\section{Description of experiment}

The experiment was made by using a driving simulator but including a test drive on public roads. The test drive on the public roads let us compare the behavior difference between the public roads and the driving simulator. However, the main part was based on the drives using the driving simulator. This was because the driving simulator could simulate many road environments and traffic scenarios. Same road environment and traffic scenarios allowed the comparison on the differences among the monitors. Furthermore, the different road environment and traffic scenarios manifest their individual influence.

Driving simulator " $\mathrm{D}^{3}$ sim" developed and provided by Mitsubishi Precision Co. Ltd. was used. The driving simulation system consists of an operation stage, three simulation computers, and four projectors. The screen is $1.5 \mathrm{~m}$ high and $2 \mathrm{~m}$ wide. Four screens are located in the front, left front, right front and right side. The data of the driving behavior and the vehicle movement are recorded at $120 \mathrm{~Hz}$.

The objective ISA modes in this study include the mandatory mode and the advisory mode. The voluntary mode was excluded because this study was an experiment in the lab so the participants could not really choose by themselves to release the function or not. Additionally, the advisory mode was designed in two ways: picture and voice. The voice information was an audible short phrase, i.e.: "speed limit is $30 \mathrm{~km} / \mathrm{h}$ "; such voice was prior recorded and played when the speed reaches to the speed limit. The picture information was shown in the upper position of the front screen as depicted in Figure 1.

The test drive routes were shown in Figure 2. Two types of trunk roads and two types of community roads were designed in terms of the general road environment conditions. The trunk roads and the community roads were separated in this study was because the different speed limits may make the drivers behave differently including the reactions for the ISA measures. 


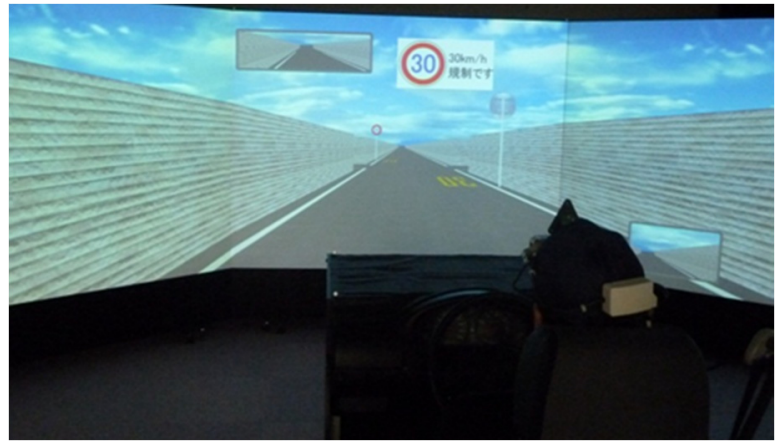

Figure 1: Situation of the experiment (example of a narrow community road).

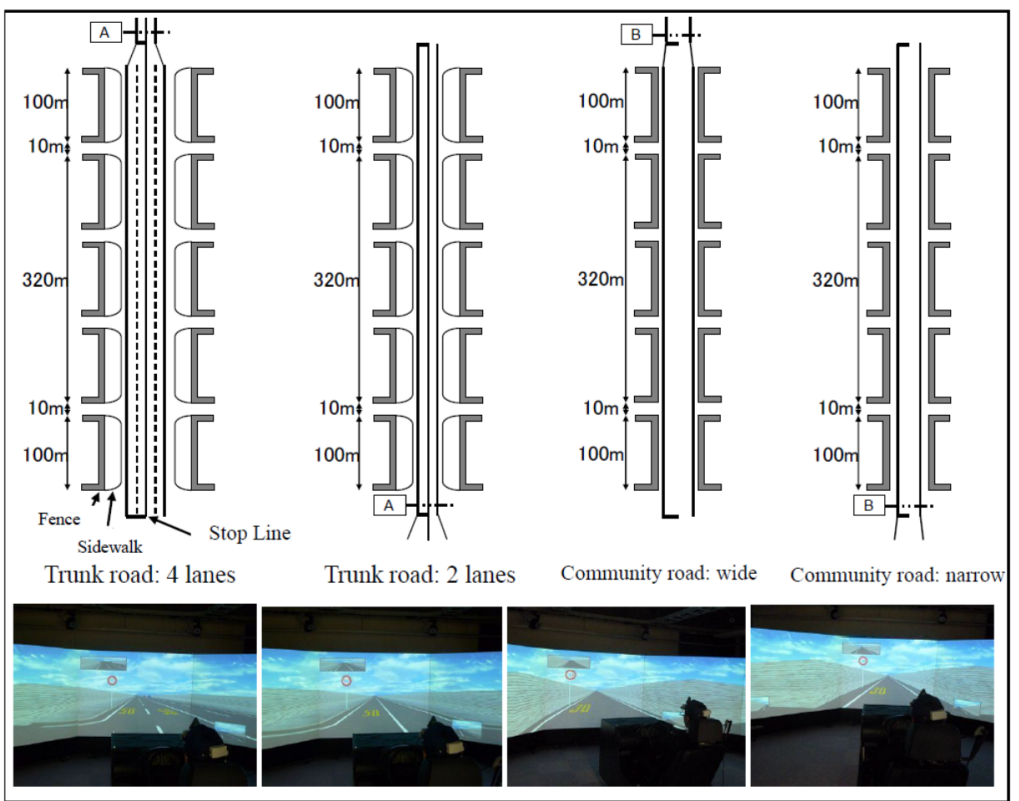

Figure 2: Objective roads used in the experiment.

In addition, two types of road environment conditions were designed here to consider the quite different lane number or widths and so on. The concrete descriptions of each type are listed in Table 1. The classes of road are in terms of the Road Structure Order of Japan. Type 4 denotes the roads locating in an urban area and Class 1 and 3 represents standards where Class 1 is the highest. During the preparation, the $40 \mathrm{~km} / \mathrm{h}$ speed limit had been discussed to be a factor for designing the test drive route, too. By considering the experiment time allocation and the workloads for the participants, the $40 \mathrm{~km} / \mathrm{h}$ speed limit was excluded for this study in order to make the experiment relatively easier to be made. 
Table 1: Parameters of the objective roads.

\begin{tabular}{|c|c|c|c|c|c|c|c|c|}
\hline & $\begin{array}{l}\text { Road } \\
\text { type }\end{array}$ & $\begin{array}{l}\text { No. of } \\
\text { lanes }\end{array}$ & $\begin{array}{l}\text { Lane } \\
\text { width }\end{array}$ & $\begin{array}{l}\text { Shoulder } \\
\text { width }\end{array}$ & $\begin{array}{l}\text { Sidewalk } \\
\text { width }\end{array}$ & $\begin{array}{l}\text { Width } \\
\text { of road }\end{array}$ & $\begin{array}{l}\text { Speed } \\
\text { limit }\end{array}$ & Class of roads \\
\hline \multirow{2}{*}{ Trunk roads } & 4-lane & 4 & 3.25 & 0.50 & 3.00 & 20.0 & \multirow{2}{*}{$50 \mathrm{~km} / \mathrm{h}$} & Type 4, Class 1 \\
\hline & 2-lane & 2 & 3.25 & 0.50 & 3.00 & 13.5 & & Type 4, Class 1 \\
\hline \multirow{2}{*}{$\begin{array}{l}\text { Community } \\
\text { roads }\end{array}$} & Wide & 1 & 5.50 & 0.50 & None & 6.50 & \multirow{2}{*}{$30 \mathrm{~km} / \mathrm{h}$} & Type 4, Class 3 \\
\hline & Narrow & 1 & 4.00 & 1.25 & None & 6.50 & & Type 4, Class 3 \\
\hline
\end{tabular}

Note: unit of width $=m$

The experiment was carried out during October through to November in 2012. 60 monitors have been recruited including 26 elderly drivers who were 65 years old and over, that are the main target age group, together with 15 adult drivers (30 through 64 years old) and 19 younger drivers (29 years old and younger) for the comparison. Excluding 8 drivers quitted the experiment because of the kinetosis, 52 monitors participates the full experiment process finally. They were 19 elder drivers (14 males and 5 females), 14 adult drivers (9 males and 5 females) and 19 younger drivers (18 males and 1 female).

The experiment was designed as consisting of the following steps:

1. The test drive is made on the public roads, then the questionnaire on the experiment.

2. The drives were made without the ISA and without the speed limit signs respective with four types of roads given in Table 1 .

3. The drives were made without the ISA but with the speed limit signs respective with the above four types of roads.

4. The drives were made with the mandatory ISA under the road environment with the speed limit signs. Then the questionnaire on the mental loads was implemented.

5. The drives were made with the advisory ISA under the road environment with the speed limit signs. Then the questionnaire on the mental loads was implemented.

6. Finally, the questionnaires about the personal attributes, the evaluation and the consciousness on the ISA were implemented.

All monitors had a 5-minutes test drive in order to get used to the driving simulator.

\section{Experiment results and discussion}

To discuss the acceptance of the ISA applications, we focus two respects: one is about the mental loads and the other is about the evaluation after the test drives.

\subsection{Analysis of influences on the mental loads}

The mental loads are expressed as 6 types: panic, uneasy, nervous, tiring, anxiously and impatient. All loads are measured by seven point scale survey: much 
agree (-3), agree (-2), a little agree (-1), no opinion (0), a little disagree (1), disagree (2) and much disagree (3). During our experiments, several monitors could not experience the ISA applications because their driving speeds did not exceed the speed limits, so that they have been excluded from the objectives of the analysis.

Figure 3 through to Figure 6 show the mental loads by the age group with respect for the mandatory ISA on the trunk roads, the mandatory ISA on the community roads, the advisory ISA on the trunk roads and the advisory ISA on the community roads.

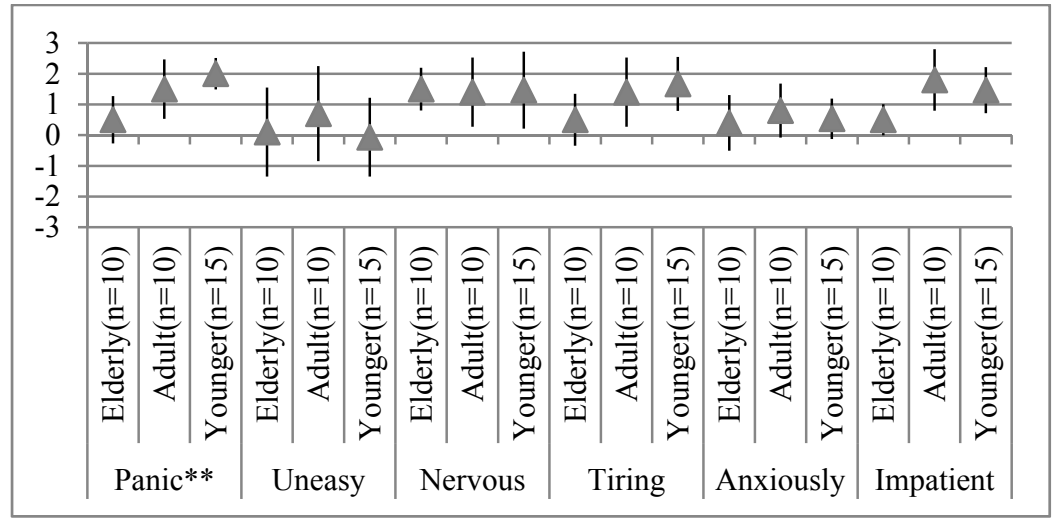

Note 1: $-3=$ much agree, $-2=$ agree, $-1=$ a little agree, $0=$ no opinion, $1=$ a little disagree, $2=$ disagree, $3=$ much disagree; Note 2: One-way analysis of variance $(*=5 \%, * *=1 \%$ significant).

Figure 3: Mental loads of the mandatory ISA on the trunk roads (the mean values and $95 \%$ confidence interval).

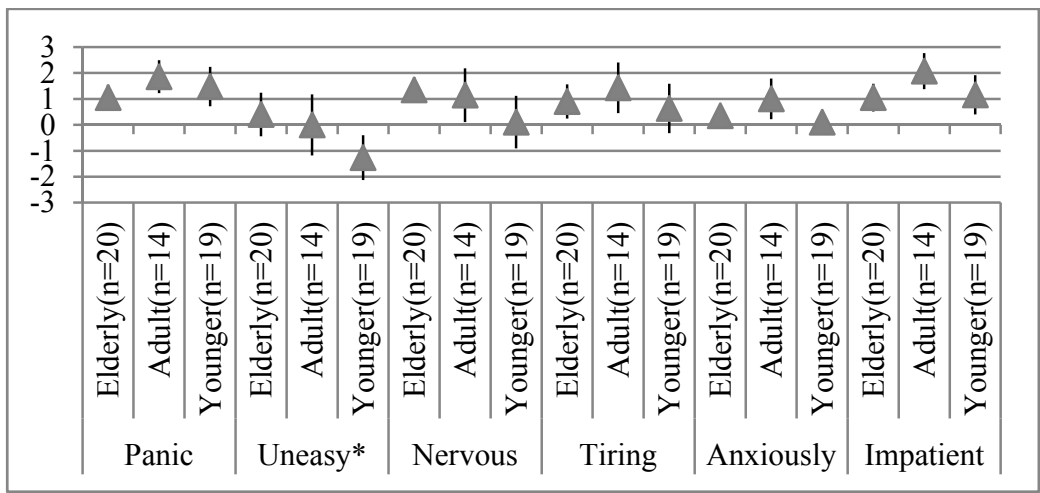

Note 1: $-3=$ much agree, $-2=$ agree, $-1=$ a little agree, $0=$ no opinion, $1=$ a little disagree, $2=$ disagree, $3=$ much disagree; Note 2 : One-way analysis of variance $(*=5 \%, * *=1 \%$ significant).

Figure 4: Mental loads of the mandatory ISA on the community roads (the mean values and $95 \%$ confidence interval). 


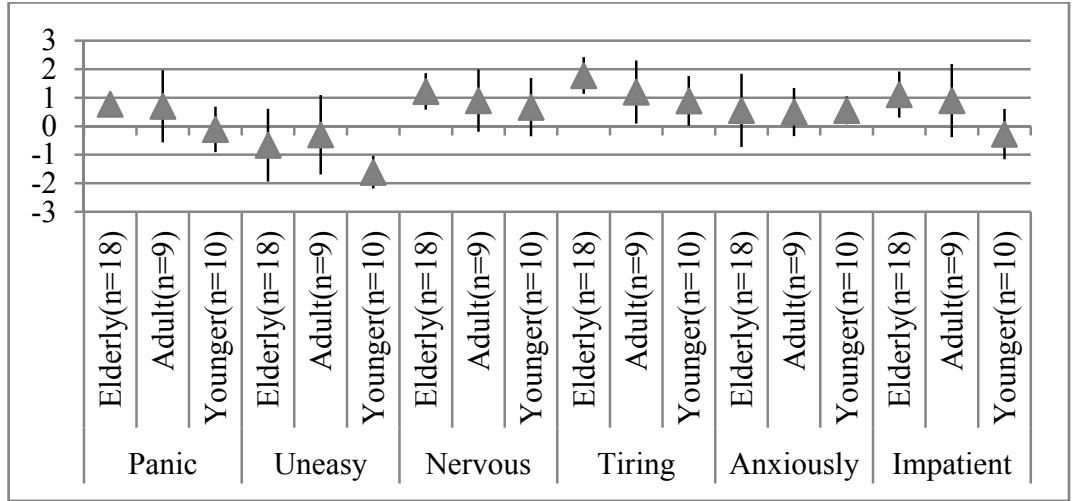

Note 1: $-3=$ much agree, $-2=$ agree, $-1=$ a little agree, $0=$ no opinion, $1=$ a little disagree, $2=$ disagree, $3=$ much disagree; Note 2 : One-way analysis of variance $(*=5 \%, * *=1 \%$ significant).

Figure 5: Mental loads of the advisory ISA on the trunk roads (the mean values and $95 \%$ confidence interval).

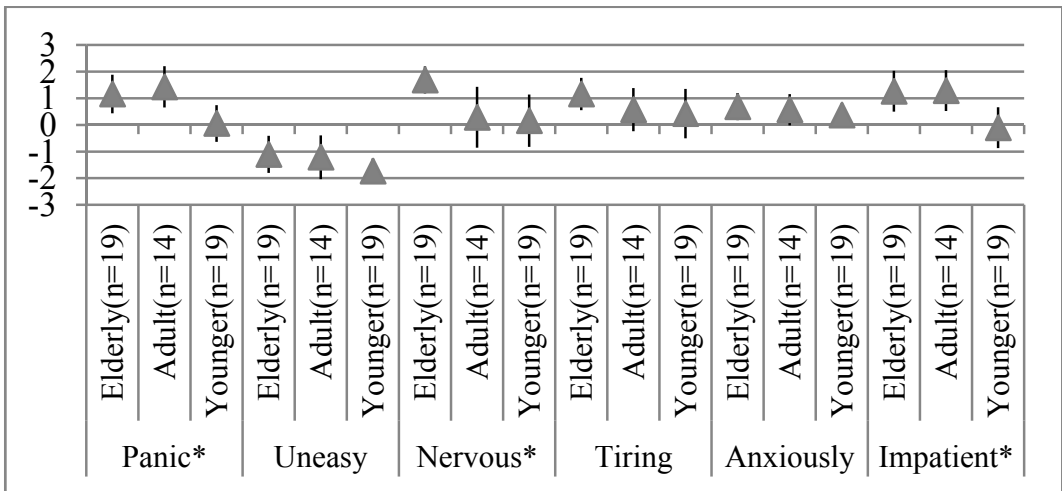

Note 1: $-3=$ much agree, $-2=$ agree, $-1=$ a little agree, $0=$ no opinion, $1=$ a little disagree, $2=$ disagree, $3=$ much disagree; Note 2 : One-way analysis of variance $(*=5 \%, * *=1 \%$ significant).

Figure 6: Mental loads of the advisory ISA on the community roads (the mean values and $95 \%$ confidence interval).

At first, on the mandatory ISA (Figures 3 and 4), all age groups tend to give positive results. Only the younger drivers show a minus value for the "uneasy" (Figure 4). Regarding the differences among the age groups, there is a statistically significant difference with the "panic" on the trunk roads in $1 \%$ level and a statistically significant difference with the "uneasy" on the community roads in $5 \%$ level. On the trunk roads, the elderly drivers have more mental loads in the viewpoint of "panic". On the community roads, the younger drivers have more mental loads in the viewpoint of "uneasy". 
Furthermore, although there are no statistically significant differences, the elderly drivers tend to have more mental loads in the viewpoints of the "tiring" and "impatient" on the trunk roads and the younger drivers tend to have more mental loads in the viewpoint of the "nervous" on the community roads. Summarizing the above, there are quite different results on the mandatory ISA by the age group. On the trunk roads, the younger drivers feel less the meatal loads and accept it very positively. On the community roads, the elderly drivers feel less the meatal loads and accept it very positively.

Now let us go to see what are about the advisory ISA applications (Figures 5 and 6). All age groups have the mental loads on both the trunk roads and the community roads in the viewpoint of the "uneasy", that is, the minus values are obtained. Regarding the differences among the age groups, there is no statistically significant difference on the trunk roads. However, in the viewpoints of "panic", "nervous" and "impatient" on the community roads, there are statistically significant differences at 5\% level. As young the drivers' age is, as more the mental loads are. Furthermore, the younger drivers show the lower values for all types of roads in all viewpoints even they may not be statistically significant. In a conclusion, the elderly drivers tend to show more positive results, that is, the elderly drivers have less mental loads especially on the community roads.

\subsection{Evaluation for the ISA services}

The evaluation for the ISA services are conducted by: 1. the reactions of the drivers when the functions work for the drivers; 2. the general evaluation for the ISA services after the drivers experienced the services; 3 . the willingness to pay when the service are put into the market; and 4. the considerations of the driver on promoting the services. All evaluations are carried out in terms of the questionnaire surveys.

Regarding the reactions of the drivers, as shown in Figure 7, the survey is based on the four point scale: 1 (completely didn't adjust the driving speed), 2 (didn't adjust the driving speed), 3 (tried to adjust the driving speed), and 4 (did best to adjust the driving speed). For the mandatory ISA, there are statistically significant differences among three age groups at $5 \%$ level. The elderly drivers tend to adjust their driving speeds much more than the adult and the younger drivers groups. However, for the advisory ISA, there is no statistically significant difference among the age groups. All drivers tend to adjust their driving speeds. The reason for the result on the mandatory ISA may be because the adult and the younger drivers understood that the system controls the driving speeds automatically, but the elderly drivers treat the system as same as the advisory ISA.

Figure 8 shows the general evaluation for the ISA services which is based on a five point scale survey: $1=$ very bad, $2=$ not good, $3=$ no opinion, $4=$ good and $5=$ very good. Comparatively, the evaluations on the advisory ISA are higher than that on the mandatory ISA. Generally, the elderly drivers have given higher evaluation than the adult and the younger drivers. Especially, there are the statistically significant differences among the age groups for the advisory ISA. 


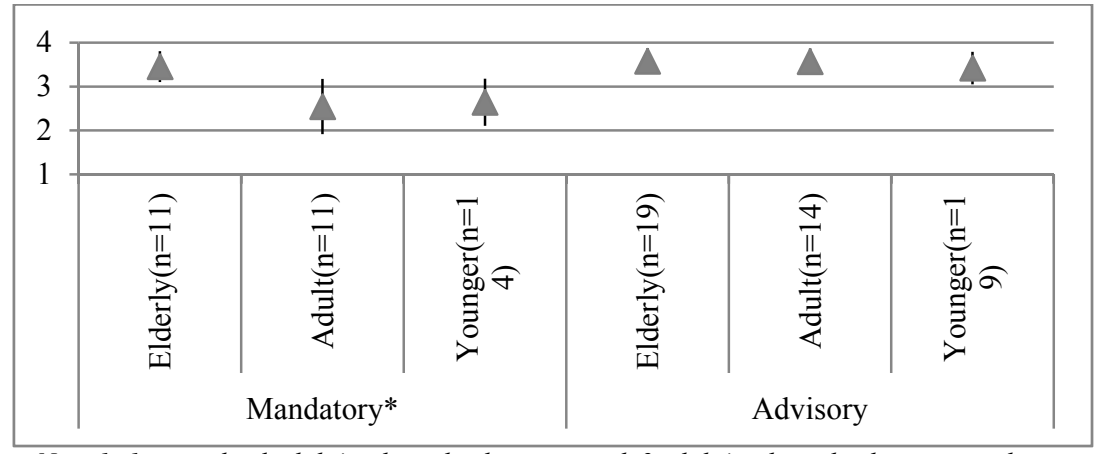

Note 1: 1=completely didn't adjust the driving speed, 2=didn't adjust the driving speed, $3=$ tried to adjust the driving speed, $4=$ did best to adjust the driving speed; Note 2: One-way analysis of variance $(*=5 \%, * *=1 \%$ significant $)$.

Figure 7: Reaction for the ISA (the mean values and 95\% confidence interval).

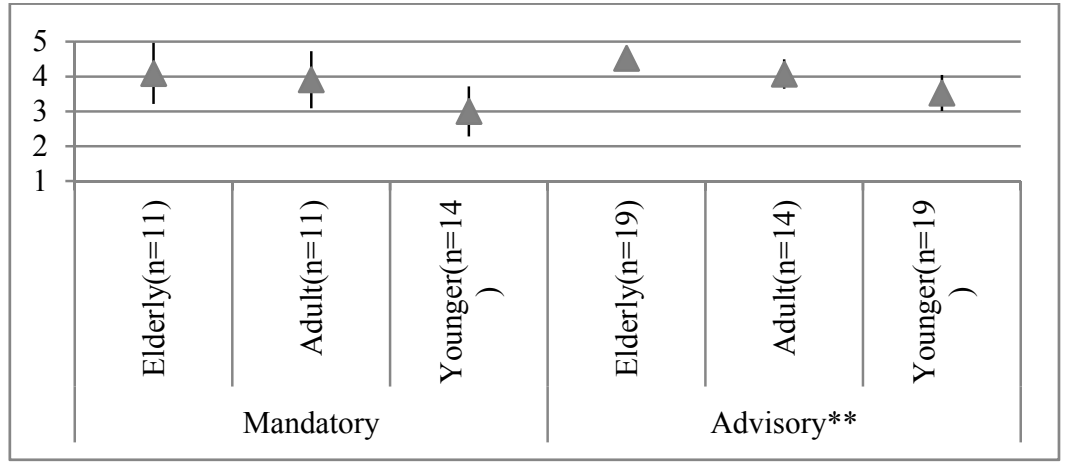

Note 1: $1=$ very bad, $2=$ not good, $3=$ no opinion, $4=$ good, $5=$ very good; Note 2: One-way analysis of variance $(*=5 \%, * *=1 \%$ significant $)$.

Figure 8: Evaluation for the ISA services (the mean values and 95\% confidence interval).

As shown in Figure 9, the willingness to pay when the service is put into the market is made by three choices in the survey: No which means "don't make use of"; Yes (without payment) which means making use of it with the condition being charge free; and Yes (with payment) which means the driver is going to make use of it even they may have to pay for it. Regarding "Yes" or "No", the advisory ISA show a larger potential than the mandatory ISA for the adult and the younger groups. However, the elderly drivers show a contrary opinion. The elder drivers like the mandatory ISA more. Then, from the viewpoint of payment, the results don't show a common sense. Furthermore, there is no statistically significant difference among the three age groups.

As for the considerations of the driver on promoting the services, the four-point scale survey has been applied by denoting $1=$ shouldn't be promoted; $2=$ not 


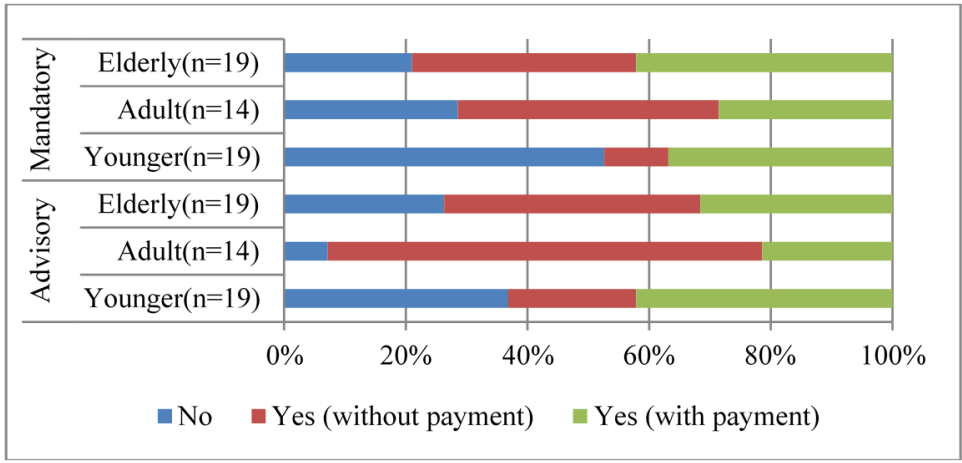

Note: Fisher's exact test (degree of freedom=4) doesn't show a statistically significant difference.

Figure 9: Market potentials of the ISA.

necessary to be promoted; $3=$ should be promoted; and $4=$ must be promoted. Figure 10 shows the results by the ISA service and by the age group. Although the younger drivers expressed a little negative consideration for the mandatory ISA service, most of drivers gave the positive consideration on promoting the ISA service for both the mandatory and advisory modes. When doing the statistical tests among the age groups, there is no significant difference in terms of the oneway analysis of variance.

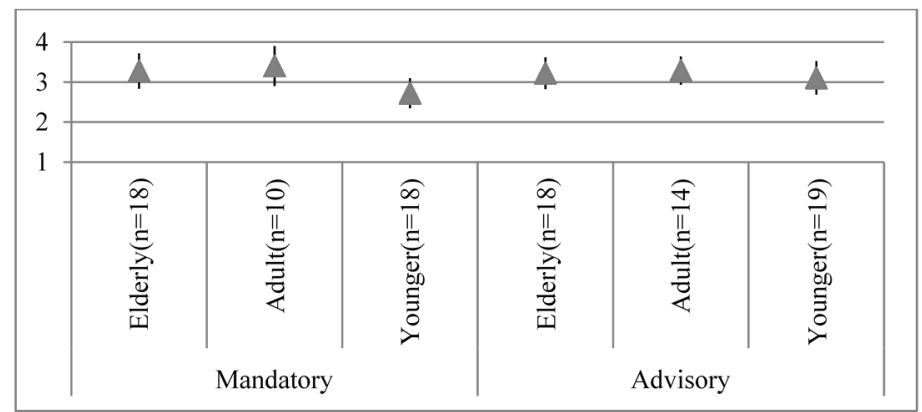

Note 1: 1=shouldn't be promoted, $2=$ not necessary to be promoted, $3=$ should be promoted, $4=$ must be promoted; Note 2: One-way analysis of variance $(*=5 \%$, $* *=1 \%$ significant).

Figure 10: Consideration for promoting ISA (the mean values and $95 \%$ confidence interval).

\section{Conclusions}

The conclusions of this study can be listed as the followings on the basis of the experiment results and discussions.

1. Regarding the acceptance in the viewpoint of mental loads, the elderly drivers have shown the different results comparing to the younger drivers for 
both mandatory and advisory ISA on the different roads. Firstly, as for the mandatory ISA, the higher evaluation is obtained from the elderly drivers on the community roads comparing to the younger drivers with less mental loads. However, the evaluation of the elderly drivers on the trunk roads is lower than the younger drivers with more mental loads. Then as for the advisory ISA, the elderly drivers show higher evaluations than the younger drivers on both the trunk roads and the community roads, that means being with less mental loads. But it is much clearly on the community roads than on the trunk roads.

2. In terms of the reactions for the ISA, regarding the mandatory ISA, the elderly drivers tend to adjust their driving speeds much more active than the younger drivers. However, there is no statistically significant difference between the elderly drivers and the younger drivers with respectively to the advisory ISA. Both the elderly drivers and the younger drivers tend to adjust their driving speeds as the reactions for the ISA.

3. Generally, the evaluations on the advisory ISA are higher than that on the mandatory ISA. Furthermore, the elderly drivers generally evaluated the ISA higher than the adult and the younger groups.

4. As for the market potentials of the ISA, there are no statistically significant differences among the different age groups or among the cost performance although the potential of the advisory ISA function seems higher than that of the mandatory ISA. Thus, we may say that the ISA has the attractiveness on its function but the benefits from the business market should not be expected too much.

5. Lastly, all age groups have considered the ISA should be promoted into the society whether the mandatory or the advisory modes.

Summarizing the above, the acceptances of the elderly drivers for both mandatory ISA and advisory ISA on the community roads are generally high. The ISA may not only make the traffic safer by reducing the driving speeds but also have the good effects to realize the traffic safety through reducing the mental loads of the driving for the elderly drivers. Comparing to the mandatory ISA, the evaluation of acceptance for the advisory ISA is higher. Thus, the promotion of the ISA introduction should be implemented from the advisory ISA. As the business market cannot be expected too much, the supports of the government and other publics are necessary and effective by considering the high social acceptance from all age groups.

As the issues to be studied further, the field test by using the cars on the roads instead of the driving simulator is necessary before making the final decision.

\section{Acknowledgements}

This study was partly supported by JSPS KAKENHI Grants Number 26540076 and a grant of Takata Foundation. Furthermore, we would like to express our sincere thanks to the cooperation provided by Mr. Obayashi, Mr. Ono and Mr. Nakatani. 


\section{References}

[1] Cabinet Office. Ninth fundamental traffic safety program. Government of Japan, 2011.

[2] WHO. Speed management - A road safety manual for decision makers and practitioners, 2008.

[3] National Policy Agency. Report on promotion of zone countermeasures for the community roads. Government of Japan, 2011.

[4] Sven Vlassenroot, Steven Broekx, Johan De Mol, Luc Int Panis, Tom Brijs, Greet Wets. Driving with intelligent speed adaptation: Final results of the Belgian ISA-trial, Transportation Research Part A, 41, pp. 267-279, 2007.

[5] Warner HW, Åberg L. The long-terms effects of an ISA speed-warning device on drivers' speeding behaviour. Transportation Research Part F; 112: pp. 96-107, 2008.

[6] Emeli Adell, András Várhelyi, Magnus Hjälmdahl. Auditory and haptic systems for in-car speed management - A comparative real life study, Transportation Research Part F, 11, 6, pp. 445-458, 2008.

[7] Committee of the Elderly Drivers' Driving Aptitude of Chubu Branch. Handbook of the elderly drivers' driving aptitude. Society of Automotive Engineers of Japan, 2005.

[8] International Association of Traffic and Safety Science. Research report on the drivers' emotional characteristics and their influences on driving behaviors, 2010.

[9] Carsten O. Is intelligent speed adaptation ready for deployment? Accident Analysis \& Prevention; 48: pp. 1-3, 2012.

[10] F. Lai, O. Carsten. What benefit does Intelligent Speed Adaptation deliver: A close examination of its effect on vehicle speeds, Accident Analysis \& Prevention, 48, pp. 4-9, 2012.

[11] H. Lahrmann, N. Agerholm, N. Tradisauskas, T. Næss, J. Juhl, L. Harms. Pay as You Speed, ISA with incentives for not speeding: A case of test driver recruitment, Accident Analysis \& Prevention, 48, 3, pp. 10-16, 2012.

[12] H. Lahrmann, N. Agerholm, N. Tradisauskas, T. Næss, J. Juhl, L. Harms. Pay as You Speed, ISA with incentives for not speeding: Results and interpretation of speed data, Accident Analysis \& Prevention, 48, 3, pp. 1728, 2012.

[13] K. Chorlton, S. Hess, S. Jamson, M. Wardman. Deal or no deal: Can incentives encourage widespread adoption of intelligent speed adaptation devices? Accident Analysis \& Prevention, 48, 3, pp. 73-82, 2012.

[14] J.W.G.M. van der Pas, V.A.W.J. Marchau, W.E. Walker, G.P. van Wee, S.H. Vlassenroot. ISA implementation and uncertainty: A literature review and expert elicitation study. Accident Analysis \& Prevention; 48: pp. 83-96, 2012.

[15] Kristie L. Young, Michael A. Regan, Thomas J. Triggs, Keren JontofHutter, Stuart Newstead. Intelligent speed adaptation - Effects and acceptance by young inexperienced drivers, Accident Analysis \& Prevention, 42, 3, pp. 935-943, 2010. 